\title{
Geovisualization Features in Substantiating Solutions in Energy Infrastructures
}

\author{
Alexander A. Izhbuldin ${ }^{1}$, Roman A. Ivanov ${ }^{2}$, Rodion Yu. Markov ${ }^{3}$ \\ Melentiev Energy Systems Institute of Siberian Branch of the Russian Academy of Sciences, \\ Lermontov str., 130 \\ Irkutsk, Russia \\ ${ }^{1}$ E-mail: izhbuldin@isem.irk.ru \\ ${ }^{2}$ E-mail: roman.and.ivanov@gmail.com \\ ${ }^{3}$ E-mail: rodion.wm@yandex.ru
}

\begin{abstract}
To justify energy solutions, it is necessary to visualize various parameters with a geospatial reference. The authors suggest visualizing the results of research in geoservices through a plug-in. Using the example of several completed projects, it is shown that this approach can be applied In connection with the disruption of the Google Earth plugin, several geoservices were compared. The rationale for selecting the Yandex geo-service and the development process for the prototype of the visualization tool are fulfilled.
\end{abstract}

Keywords: geovisualization, geoservices, energy spatial tasks, yandex.maps.

\section{Introduction}

Energy infrastructures are geographically distributed, connected or unrelated objects. For the study of energy infrastructures, it is necessary to prepare and structure data reflecting the main technical characteristics of the objects under consideration, for example: the power of energy sources, the capacity of transport structures, the type of fuel, the length of power transmission lines, etc. An important component of studies of geographically distributed objects is the analysis of the relationships between them. The tool for such studies are popular geoservices. Similar works for the mapping of geospatial information in solving problems of the development of energy systems using GIS and geoservices are conducted in various institutes of the Russian Academy of Sciences. They are associated with mapping research results on the renewable energy resource potential [1,2], environmental and energy security [3].

\section{Tools and completed projects}

In the Melentiev Energy Systems Institute of Siberian Branch of Russian Academy of Sciences developed a web-service Geocomponent for visualization of geospatial information by means of geoservices [4]. For dynamic visualization and geocoding, the Google Earth plugin was built into the web service (fig. 1).

The geocomponent was used to solve a number of geospatial tasks using geovisualization, such as:

1) obtaining a profile of relief and length of sections with various mining and geological conditions of construction for the initial economic justification of pipelines, power lines and highways [5];

2) determining the contribution of various energy objects to environmental pollution by visualization of emission fields and distribution functions of pollutants [6];

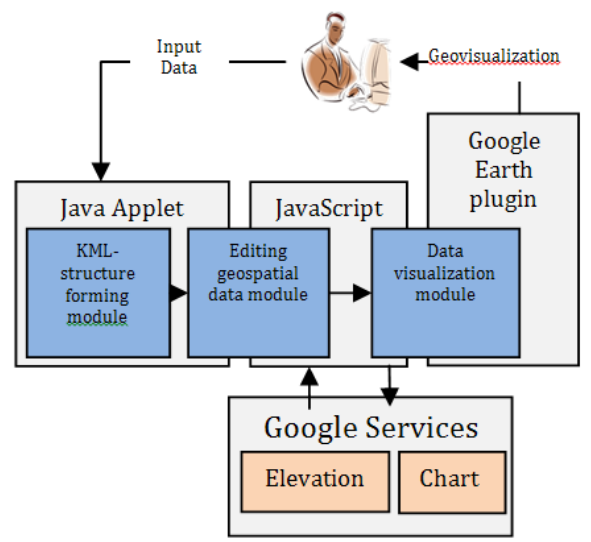

Fig. 1. Web-service Architecture. 
3) displaying the current state of energy security both in Russia as a whole and in individual federal districts through the display of energy security indicators in relation to the territories. [7].

4)identifying energy facilities and visualizing the main characteristics of the ecological state in zones of special nature use, using the example of the central ecological zone of the Baikal natural territory $[8,9]$.

The initial data for visualization is presented in the file consisting of a set of tables. More often than not, each table contains data combined by geographical location (federal district, region, district).

To display and analyze an indicator, you must select the cell of one table and show the value of this indicator from the other tables. This mapping will allow you to visually compare one parameter that is in different tables in the source file. It is assumed that such a cartography mapping will simplify the analysis of information and obtain a new quality of data representation.

In connection with the termination in 2016 of the Google Earth plug-in $[10,11]$, there was a need to find and select an alternative geo-service. The required geoservice must meet certain basic requirements, for example, the presence of a plug-in with the ability to access through the API and support the $\mathrm{Kml}$ format for markup of geospatial information. In Table. 1 shows a comparison of the three geoservices of Google, Arcgis Earth and Yandex.

Table 1. Comparison of the geoservices advantages.

\begin{tabular}{|l|c|c|c|}
\hline Criteria & $\begin{array}{l}\text { Google } \\
\text { Earth }\end{array}$ & $\begin{array}{l}\text { Yandex } \\
\text { Maps }\end{array}$ & $\begin{array}{l}\text { Arcgis } \\
\text { Earth }\end{array}$ \\
\hline Multiscale & + & + & + \\
\hline Multilayer & + & + & + \\
\hline Multiangularity & + & - & + \\
\hline Kml format support & + & + & + \\
\hline API \& plugin & - & + & - \\
\hline $\begin{array}{l}\text { Ability to modify } \\
\text { modules }\end{array}$ & - & + & - \\
\hline Technical support & $+/-$ & + & - \\
\hline
\end{tabular}

The table below examined the criteria that are fundamental to the interaction with these services in order to enable the application of a new approach and the use of tools for dealing with geospatial information in research and decision-making in the energy sector.
It is worth noting that Yandex service has nearly all the necessary technology inherent geoservices. And the availability of mechanisms for modifying modules and high-quality technical support allow it to outpace the geoservice Google Earth.

The main disadvantage of the Yandex.maps technological functionality, in contrast to the geoservice of Google, is the lack of competitiveness, i.e. the ability to display information in three-dimensional space Thus, Yandex.maps service satisfies the most advanced criteria and can be used as an alternative to Google geoservices.

ArcGis Earth geoservice also offers threedimensional visualization, but it does not have a plug-in, and accordingly there is no possibility of dynamic visualization and geocoding.

Applying the Yandex.Maps service entails adapting the architecture of the Geocomponent tool.

\section{Adaptation of the architecture of the visualization tool}

To adapt the architecture, the developer documentation of the Yandex.maps API was studied and the main limitations and nuances were revealed.

Yandex.Maps do not support a folded structure in the KML file. In order to allow the connection of separate layers, it is necessary to divide one KML file into several parts and load them separately. In addition, the styles of displaying objects in KML-files are supported by Yandex.maps only partially. For example, the color and size of the labels may not be displayed correctly. If the stylesheet specifies a picture, as an object's display, then you need to make sure that it is in a direct link (http with public access). This is due to the fact that KML processing is performed on the side of Yandex servers, and the final version of the map with objects is transferred to client part of software for visualization.

Also, there is a need to develop additional functions for interpreting and displaying tabular data. In particular, there are a number of tasks in which data are presented as tables in XLS or HTML formats. It is necessary to process such files and build a visualization based on the data from these tables.

It is suggested to use multidimensional arrays as the main data structure. An important aspect, when using such arrays, is the definition of the slice of the data to display. Eventually, the user determines what kind of slice, and on what data he needs to output. In a multidimensional array, you can enter the information of any kind and dynamically add the necessary data, for example, geographic coordinates. The new architecture, 
taking into account all the features described above, is presented in the figure 2 .

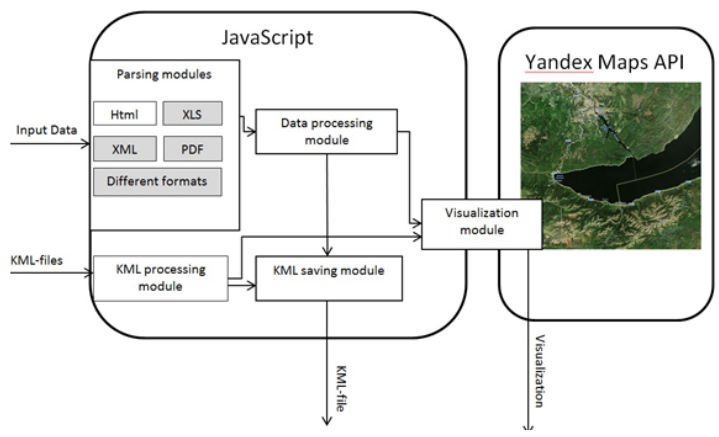

Fig. 2. The architecture of the developed web application.

This architecture assumes the use of the JavaScript language as it is written on the Yandex.Maps API.

The system consists of several modules:

- Parsing modules

- Data processing module

- KML processing module

- KML Forming module

- Visualization module

Modules of parsing for various formats are necessary in order to automatically collect the required data from the source files and transfer them to the processing. For example, HTML or XLS files containing several tables of the same type.

The data processing module creates arrays from the extracted data to display. Such arrays must be multidimensional, depending on the dimensionality of the extracted data.

The KML processing module is required to import and process KML files. Features of the display in the Yandex.Maps service of files with a structure and several styles require additional processing for the correct display.

The KML Forming module creates a KML-file that contains the data displayed in the plugin. It can be reloaded again, or imported into other geoservices, such as the desktop Google Earth application. Thus, spatial problems that require a three-dimensional mapping can be solved by visualizing the $\mathrm{kml}$ file.

The visualization module is necessary for preparing and loading the processed data in the Yandex.Maps API.

Based on the proposed architecture, a research prototype was developed that implements the basic functions.
The algorithm of the developed application for a specific task is as follows:

1) the user loads the input data;

2) the parsing module extracts from the source file a set of two-dimensional arrays (tables) and passes them to the processing module $\mathrm{c}$ indicating the names of columns, rows and tables;

3) the processing module forms a three-dimensional associative array in which the slices correspond to the names of the transmitted columns, rows, and tables (figure 3);

4) the user selects the desired slices;

5) the processing module sends the data selected in this slices to the visualization module;

6) the visualization module displays the selected data on the map.

An example of visualization of the selected indicator (mining of natural gas) for each federal district of the Russian Federation is presented in figure 4. Also for each object you can display additional information if necessary.

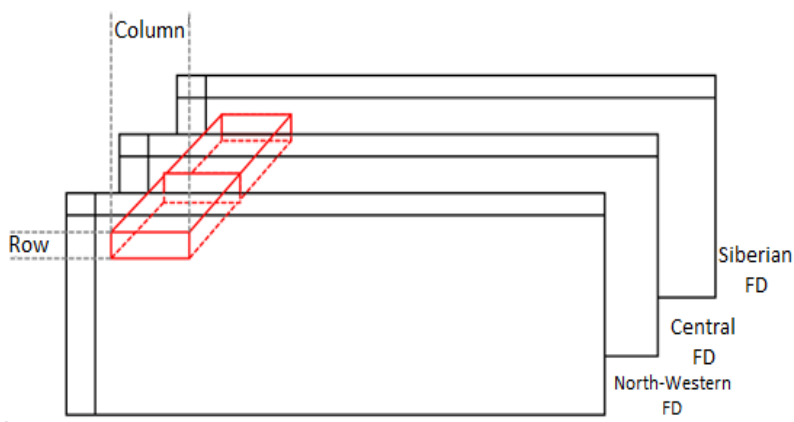

Fig. 3. Visual representation of a three-dimensional array.

\section{Conclusion}

In connection with the need to visualize geospatial information in the tasks of assessing the state and development of energy systems, researchers use different approaches. Visualization using geoservices can successfully solve different classes of spatial tasks in energy. The authors proposed the adaptation of the instrument of visualization, taking into account the use of the plugin geo-service Google. The use of Yandex geoservice is proposed and the architecture of the tool is designed. 

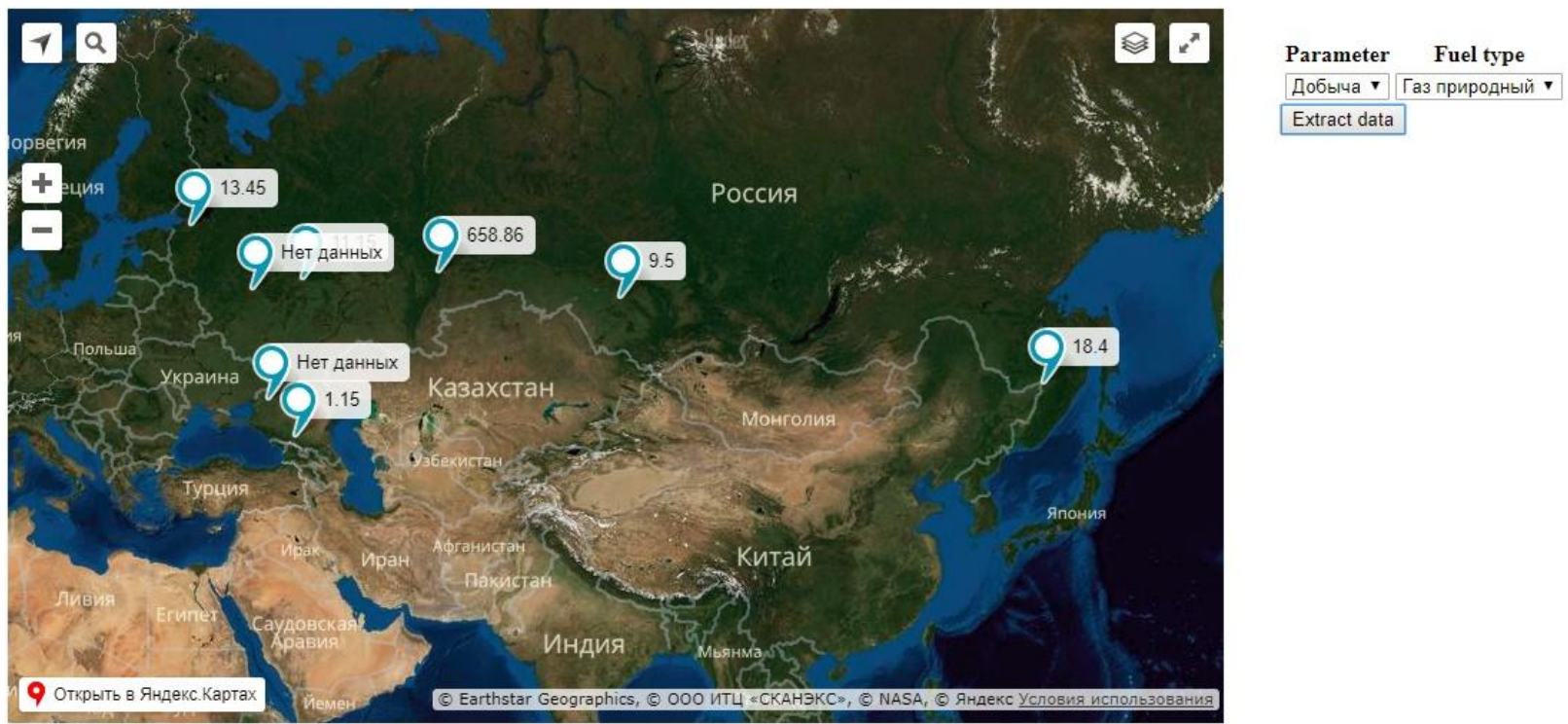

Fig. 4. Visualization of the values of the indicator for each federal district of the Russian Federation by Yandex.Maps.

\section{Acknowledgements}

This work is partially supported by the RFBR grants №16-07-00474a, №18-57-81001a, №17-07-01341a, №18-07-00714a.

\section{References}

1. Popel O.S., Frid S.E., Kiseleva S.V., Kolomiets Yu.G., Terekhova E.N. Distribution of energy resources of solar radiation across Russia, Energy: economics, technology, ecology, № 1. (2007) 15-23.

2. Oshchepkova Ya. O., Kiushkina V. R., Cluster Analysis of the Potential of Renewable Energy Sources in the Republic of Sakha (Yakutia), Internet Journal "Naukovedenie", №4 (23). (2014) 12-17.

3. Martynyuk P. P., Kiushkina V. R., Autonomous energy sources in the environmental safety of the North, Energetika. Innovative directions in the energy sector. $C A L S$-technologies in power engineering, Vol. 1. (2016) 26-33.

4. Massel L. V., Ivanov R. A., Chemezov A. A., Webapplication for 3D-visualization in research and substantiation of solutions in the energy sector, Educational resources and technologies, No. 5 (8). (2014) 101-107.

5. Izhbuldin A.K., Ivanov R.A. Application of geoservices for the tasks of comparative express assessment of oil and gas pipeline routes, Problems of Economics and Management of the Oil and Gas Complex, No. 12. (2015) 21-28

6. Kuchmenko E. V., Zarodnyuk M S., Ivanov R. A., Chipanina E. V., Modeling the anthropogenic impact of industrial centers of the Irkutsk region on the southern basin of the Lake Baikal, in Proc. of the international scientific conference "Intelligent decision-making systems and problems of computing intelligence". (Ukraine, Kherson: KHNTU, 2013) pp. 135-137.

7. Energy security in Russia: problems and solutions / Pyatkova N. I., Rabchuk V. I., Senderov S. M., Slavin G. B., Cheltsov M. B. (Novosibirsk: Siberian Branch of the Russian Academy of Sciences, 2011), 198 p. ISBN 9785-7692-1163-8.

8. Ivanova I. Yu., Ivanov R. A., The use of geovisualization in the analysis of the location of energy infrastructure objects of the central ecological zone of the Baikal natural territory, Information and mathematical technologies in science and management, № 4-2. (2016) 80-89.

9. Ivanova I. Yu., Ivanov R. A., Geovisualization and visual analytics on the example of the substantiation of the development of the energy infrastructure of the central ecological zone of the Baikal natural territory, Informational and mathematical technologies in science and management. №1. (2018) 87-98.

10. Developers of the Google Earth plugin and API https://developers.google.com/earth/

11. Announcing deprecation of the Google Earth API https://mapsplatform.googleblog.com/2014/12/announcin g-deprecation-of-google-earth.html 